Dom. Cien., ISSN: 2477-8818

Vol. 4, núm.3., jul, 2018, pp. 360-391

\title{
La Vinculación como estrategia de la Institución de Educación Superior
}

\section{Linking as a strategy of the Higher Education Institution}

\section{Vinculação como estratégia da Instituição de Ensino Superior}

\author{
Verónica R. Indacochea-González I \\ verónica.inn@gmail.com \\ Carmen A. Álvarez-Vásquez II \\ carmen.alvarez@gmail.com \\ José M. Piguave-Reyes ${ }^{\text {III }}$ \\ josepiguaves@hotmail.com
}

\author{
Marlon A. Cañarte-Chele IV \\ marlon_12ca@hotmail.com \\ Virginia E. Pincay-Pin V \\ virginia_966pi@gmail.com \\ Rosa del Rocío Pinargote-Chancay VI \\ rosa_rocio52@gmail.com
}

Recibido: 27 de febrero de 2018 * Corregido: 18 de mayo de 2018 * Aceptado: 09 de julio de 2018

\footnotetext{
Licenciada en Enfermería, Hospital de Especialidades Portoviejo, Portoviejo, Ecuador.

II. Ingeniera Comercial Mg. DUIE, Docente UNESUM, Jipijapa; Ecuador.

III. Licenciado en Laboratorio Clínico, Mg. en Epidemiología, Centro Especializado en Diagnóstico y Tratamiento Muñoz, Ecuador.

Iv. Médico Cirujano, Especialista en Cardiología, Docente UNESUM, Jipijapa; Ecuador.

v. Licenciada en Enfermería, Mg. Gerencia en Salud para el Desarrollo Local, Docente UNESUM, Jipijapa; Ecuador.

vI. Licenciada en Enfermería, Mg. en Epidemiología, Docente UNESUN, Jipijapa; Ecuador.
} 


\section{Resumen}

El objetivo de este artículo es explorar un acercamiento sobre la vinculación como estrategia de la Institución de Educación Superior (IES), la vinculación como estrategia de la formación de competencias profesionales, las universidades tienen un rol importante que jugar puesto que son ellas las que forman a los científicos, profesionales y líderes que decidirán de las estrategias económicas y públicas nacionales e internacionales. La vinculación con la comunidad como estrategia de desarrollo, un aspecto muy importante de las políticas de desarrollo a nivel nacional que durante los últimos años se han impulsado diversas acciones tendientes a reforzar estas actividades, La vinculación con la sociedad y los sectores productivos apoyado por los sectores estatales considerando la importancia de la ciencia, la tecnología y la innovación, los tutores e investigadores han de elaborar estrategias y propender tareas para establecer relaciones de vinculación con el sector productivo; se logre incorporar programas, con una estrategia general de intervención que le permita plasmar en muchas prácticas educativas hacia las necesidades de la realidad social y productiva, todo esto en el ámbito en que se desenvuelve la universidad con los sectores sociales, productores y estudiantes en formación profesional, que la universidad investigue la necesidad de vinculación que tiene con el entorno globalizado.

Palabras claves: Vinculación como estrategia, Institución de Educación Superior, la comunidad, los tutores e investigadores, los sectores productivos. 


\begin{abstract}
The objective of this article is to explore an approach to linkage as a strategy of the Institution of Higher Education (IES), the linkage as a strategy for the formation of professional skills, universities have an important role to play since they are the ones that form to scientists, professionals and leaders who will decide on national and international economic and public strategies. The link with the community as a development strategy, a very important aspect of the development policies at the national level that during the last years have been promoted various actions tending to reinforce these activities, The link with the society and the productive sectors supported by the State sectors considering the importance of science, technology and innovation, tutors and researchers have to develop strategies and assign tasks to establish relations of linkage with the productive sector; it is possible to incorporate programs, with a general strategy of intervention that allows it to translate into many educational practices towards the needs of the social and productive reality, all this in the field in which the university develops with the social sectors, producers and students in training professional, that the university investigates the need for links with theglobalized environment.
\end{abstract}

Key words: Linking as a strategy, Higher Education Institution, the community, the tutors and researchers, the productive sectors. 


\section{Introducción.}

La institución de educación superior del siglo XXI despertó el interés por la competencia dando prioridad a las respuestas de los requerimientos mediatos e inmediatos, la globalización es al mismo tiempo un proceso cultural y educativo en la nueva sociedad del conocimiento, fundamentalmente las instituciones están obligadas a permanentes cambios e innovaciones científicas y tecnológicas, modificando trascendentalmente las funciones de la universidad.

La educación superior en la actualidad frente a los procesos de globalización es evidente que difunde la transformación de sus estándares organizacionales y su estructura académica, con el firme propósito de generar una mejor calidad educativa, la misma que debe estar alineada a disputar en un mercado cada vez más flexible sea este mundial, regional o local; la universidad a reformulado sus estrategias educativas, cumpliendo con numerosas reformas estructurales y generando diferentes programas estratégicos de desarrollo.

Justo en un tiempo histórico que investiga la humanización y la inserción de todos y todas, la institución de educación superior que inseparablemente logre impulsar el diálogo constructivo entre los diversos intereses de la sociedad civil y las ciencias para el aseguramiento de un discernimiento socialmente solidario. Se prevee que la educación superior tendrá un incremento significativo en cuanto al ingreso de estudiantes durante las próximas décadas.

El futuro de la IES esta en la capacidad que tengan para considerar así misma e innovar su participación ante una sociedad rápidamente variable. En esta ruta, el gran reto para la institución de educación superior es doble. Por una parte, debe dar respuesta a los retos que la sociedad plantea y cada vez se vuelve más compleja, que demanda mayor eficiencia en la formación de sus ciudadanos

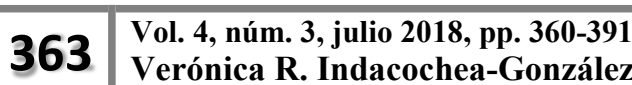
Verónica R. Indacochea-González; Carmen A. Álvarez-Vásquez; José M. Piguave-Reyes; Marlon A. Cañarte-Chele; Virginia E. Pincay-Pin; Rosa del Rocío Pinargote-Chancay
} 
y que, al mismo tiempo, reclama un régimen universitario de calidad que garantice el derecho a la igualdad de oportunidades particularmente a la IES pública.

Por lo que no puede dejar de lado algunas de sus misiones como es el de adoptar un proceso convertidor capaz de activar los recursos y la capacidad de recapacitar y actuar sin fragmentos anteriores de los personajes afectados por varias formas de exclusión. La Investigación Acción Participativa (IAP), destinada al trabajo social y al desarrollo comunitario, responde a este "replanteamiento" de la política social.

Esta estrategia se aconseja en proyectos de carácter social y el trabajo en conjunto con las comunidades, pues son necesarias tanto para el empoderamiento de éstas hacia su patrimonio común, como para el cambio cognoscitivo y estructural de los estudiantes que salen del salón de clase. Así, la experiencia de vinculación se convierte en un medio de enlace entre la academia y la cultura popular. Esta intervención transforma su entorno marcando su desarrollo, con un profundo compromiso ético con la comunidad, la modernización e introducción de nuevas tecnologías en el sistema productivo ayudará a construir una sociedad más justa.

Otra gran estrategia es el grado de armonía e integración así como el comportamiento de los aspectos gubernamental, social y empresarial y del académico-investigador de la IES, así como de los Organismos Públicos y ONG's.; la solución para el éxito o el fracaso de la propuesta de la “tercera misión”, como nueva configuración de la función de la universidad en la actual sociedad del conocimiento.

Esta unificación y cooperación responde a la alusión, antes citada, del "modelo de triple hélice", en la que el giro coordinado de cada una de las palas que representan los agentes que 
protagonizan los actuales Sistemas de Ciencia, Tecnología y sociedad, es decir, universidades, gobiernos y empresas, con esta habilidad se logre generar la energía necesario para que el procedimiento de innovación despegue y se eleve lo más alto posible.

Las instituciones de educación superior ante un mundo en constante proceso de cambio, aparece la educación permanente como respuesta pedagógica estratégica, que hace de la educación asunto de toda la vida y concede a los educandos de los instrumentos intelectuales que le permiten "aprender a aprender" y adaptarse a los nuevos requerimientos del mundo social y laboral, con el propósito de alcanzar los objetivos la universidad, la enseñanza se hace cada día más compleja y el aprender es una experiencia que en este caso se trata del "aprender haciendo", lo cual es un reto para el estudiante universitario, el mismo que debe responder a las necesidades educativas y sociales.

Las IES deben configurar su oferta de servicios en coherencia con los programas institucionales de vinculación, reconocer las necesidades de los sectores sociales y productivos. Además, se deberá superar la concepción de la vinculación como un camino de una sola vía por lo que los tutores están obligados a elaborar los sílabos, planificar, organizar, gestionar e implementar en el aula o en el área procesos de enseñanza-aprendizaje, ejercicios que establecen de acuerdo con su formación, experiencia, así como con los recursos con que dispone en el contexto donde se desenvuelven.

Debe ser la arena en la cual se desarrolla culturalmente una nación e incluir en la formación profesional de sus alumnos valores y directrices éticas que alienten el compromiso social, mismo que se adquiere al ser beneficiado por la sociedad al poder estudiar, constantemente compartir buenas prácticas universitarias posibles de ser replicadas por otras universidades, donde el establecimiento de la vinculación como el vínculo permanente de la academia con los sectores

\footnotetext{
365 Vol. 4, núm. 3, julio 2018, pp. 360-391 Verónica R. Indacochea-González; Carmen A. Álvarez-Vásquez; José M. Piguave-Reyes; Marlon A. Cañarte-Chele; Virginia E. Pincay-Pin; Rosa del Rocío Pinargote-Chancay
} 
comunitarios, productivos, culturales e instituciones, se active en función de generar el conocimiento mediante la metodología de aprender y enseñar, que, hacia adentro, fortalece el perfil de los futuros profesionales, atesora el acervo del tutor o docente y hacia fuera contribuye a la solución de los problemas educativos y sociales.

\section{Desarrollo}

La vinculación como estrategia de la formación de competencias profesionales

La estrategia propuesta para el perfeccionamiento de la formación de competencias profesionales considera acciones relacionadas con el diagnóstico de la inserción laboral de graduados y percepción del mercado laboral sobre sus competencias profesionales, rediseño curricular de la carrera administración de Empresas. (Perfil del egresado, competencias, malla curricular y proyectos de investigación de la carrera) y perfeccionamiento de los mecanismos de vinculación universidad empresa. (Componente laboral investigativo, pertinencia e inserción laboral de los graduados). Álvarez, Romero, Murillo, (2014).

Es falso pensar en formarse haciendo solamente. La experiencia de un trabajo profesional no puede ser formadora para aquel que la lleva a cabo, salvo si encuentran los medios de volver, de rever lo que ha hecho, de hacer un balance reflexivo. Reflexionarse el mismo al tiempo de reflejar y tratar de comprender, y en ese momento sí hay formación. Ferry (1997).

La formación y la acción tienen que ir de la mano para lograr un desarrollo verdaderamente justo y sostenible, y las universidades tienen un papel fundamental que jugar en ese sentido, puesto que son ellas las que forman a los científicos, profesionales y líderes que decidirán de las políticas económicas y públicas nacionales e internacionales. Estamos afrontando un momento histórico que

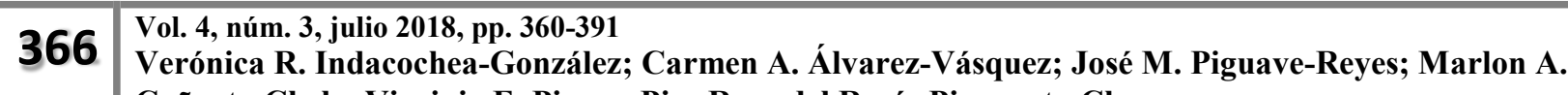
Cañarte-Chele; Virginia E. Pincay-Pin; Rosa del Rocío Pinargote-Chancay
} 
busca la rehumanización y la inclusión de todos y todas, y las Universidades y la Educación Superior toda puede y debe fomentar un diálogo constructivo entre los diferentes intereses de la sociedad civil y las ciencias para la cocreación de un conocimiento socialmente responsable. Grimaldo, (2018).

La ausencia de memoria histórica conlleva perjuicios insoslayables para el presente y futuro de las naciones. La necesidad de la reforma universitaria en Latinoamérica permanece latente. Acudir al estudio del Manifiesto de Córdoba de 1918 es pertinente. La apertura universitaria a las capas sociales emergentes, el énfasis de su misión social, la autonomía son desarrollos insuficientes ante la gran demanda de jóvenes por no poderse formar a nivel educativo terciario para lograr aportar a la transformación de su vida personal, familiar y comunitaria, Parra de Marroquín, (2018).

Morán (1993); Ferry (1997); Sánchez (1998); Barabtarlo (2002); Yurén (2005); Navia (2006); Costa (2012), los autores antes indicados coinciden en manifestar que la formación es en principio, una reflexión sobre sí mismo, tomar conciencia de nuestro ser y nuestro tiempo, reconocer nuestras debilidades y fortalezas ante las tareas y exigencias sean estas profesionales o personales.

Pereda, C., De Prada M. Á., y Actis, W., (2003), se ha demostrado en la práctica, tal actitud se convierte en motor de un proceso transformador capaz de movilizar los recursos y la capacidad de pensar y actuar - sin recortes previos - de las personas afectadas por las diversas formas de exclusión. La Investigación Acción Participativa (IAP), aplicada al trabajo social y al desarrollo comunitario, responde a este "replanteamiento" de la política social. La IAP no es una técnica que se pueda incorporar en el programa, sino un enfoque o estrategia general de intervención que se puede plasmar en muchas prácticas concretas que dependerán de la creatividad de los protagonistas y de las circunstancias presentes en cada caso. 
Costa, (2011), manifiesta que "la educación tiende a contribuir para la concientización de los profesores y en el desenvolvimiento de su sensibilidad".

La educación es asumida como un proceso más complejo que implica el compromiso responsable de varios actores, estudiantes, profesores, padres de familia, personal directivo y administrativo, en suma la escuela, en donde se lleva a cabo este proceso pero también la comunidad a la cual pertenece y para fijar de alguna manera los límites de esta ubicación, a la sociedad de la cual forman parte de estos componentes. Briones, (1999).

Tünnermann, (2011), la responsabilidad social universitaria contribuye a clarificar y fortalecer la relación Universidad-Sociedad. Las cuatro funciones universitarias: gestión, docencia, investigación y extensión, cuando son ejercidas con una perspectiva ética, contribuyen a poner de manifiesto esa responsabilidad, de la que deben ser protagonistas todos los estamentos universitarios: directivos, administradores, docentes, investigadores, extensionistas y, principalmente, los estudiantes. Consecuente con este criterio y de la necesidad de una integración creativa Universidad-Sociedad, existe la posibilidad de hacer de la responsabilidad social el eje de la acción universitaria.

La educación es a la vez un mundo en sí mismo y un reflejo del mundo. Está sometida a la sociedad y concurre a sus fines, especialmente al desarrollo de sus fuerzas productivas, atendiendo a la renovación de sus recursos, de forma más general reacciona necesariamente, aunque sea solo por el conocimiento que de ello proporciona, a las condiciones ambientales a las que se haya sometida. Por eso mismo, contribuye a engendrar las condiciones objetivas de su propia transformación de su propio progreso. En el plano de lo vivo, tal como lo sienten enseñantes, enseñados y padres, la función social de la práctica educativa, bajo las formas pedagógicas y en los contextos múltiples 
donde se ejerce, aparece como infinitamente compleja, y se comprueba tanto la magnitud del poder liberador de la educación como sus impotencias, sus deficiencias y sus coerciones, UNESCO, citado por Briones, (1999).

Los países de Iberoamérica deben aplicar políticas que conduzcan a impulsar la innovación, crear e incorporar conocimiento científico y tecnológico de calidad y socialmente relevante, difundirlo y aplicarlo en las empresas y otras instituciones. En este proceso deben colaborar, tanto los investigadores, los tecnólogos y los gestores, como los empresarios, los gobiernos, las instituciones educativas, la opinión pública y otros actores de la sociedad. Todos ellos han de estar involucrados en un proceso de aprendizaje que requiere cambios profundos de mentalidad y de conductas. OEI, (2012).

Las universidades latinoamericanas ejecutan el 36,6\% de la I+D regional, mientras que, si se tiene en cuenta a iberoamericanas en la ejecución de la investigación científica y el desarrollo tecnológico con el promedio de los países de la Organización para la Cooperación y el Desarrollo Económico (OCDE) (17\%), Estados Unidos (14,3\%), la Unión Europea (UE-27) (22,1\%), queda de manifiesto el papel protagónico que tienen las universidades en la investigación científica delos países de la región. OEI, (2012).

Lamentablemente las universidades asignan muy pocos recursos para la realización de investigaciones, algunos esfuerzos se hacen por la vía de consultorías puntuales que solicitan la cooperación internacional. Oseguera, (2005).

Alcántar y Arcos, (2004), expresan que los cambios en la economía, los nuevos liderazgos militares y económicos, las propuestas de nuevos paradigmas que ha traído consigo la globalización, 
han motivado transformaciones sociales que han impactado a la educación superior. Ello, aunado a la escasez de recursos financieros de los gobiernos para apoyar a la educación; los cambios tecnológicos y de modelos educativos basados en el aprendizaje a lo largo de toda la vida; un mundo del trabajo más diversificado, con mayor competencia y nuevos requerimientos en destrezas, habilidades y conocimientos, ha obligado a las IES a buscar nuevas estrategias para responder adecuadamente a estos retos.

En la sociedad del conocimiento nadie puede sobrevivir solo. La cooperación entre actores públicos y privados, universidades y organizaciones de la sociedad civil es clave para generar nuevas ideas y tecnologías. Sin embargo, las diferencias persisten entre las distintas regiones del mundo ya que mientras en la OCDE existen 3651 investigadores por cada millón de habitantes, en América Latina existen solo 495. En parte por ello, Norteamérica y los países de Europa que pertenecen a la OCDE producen casi 20 veces más artículos y 40 veces más citas vinculadas con ciencia y tecnología que los países latinoamericanos. Bellettini, (2012).

Estas iniciativas tienen en común el interés metodológico por favorecer la participación de los afectados en los procesos de investigación-acción. Sin embargo, parten de estrategias y planteamientos teóricos diversos $\mathrm{y}$, en general, su nivel de instrumentación técnica está poco desarrollado. Las diferencias en el plano estratégico (el para qué y el para quiénes de la acción emprendida) dependen de la demanda: quiénes son los sujetos reales de los procesos puestos en marcha y qué fines o efectos sociales persiguen a través de ellos. Estas diferencias a nivel estratégico se traducen-o disfrazan-recurriendo a aquellas tradiciones teóricas. Pereda, C., De Prada M. Á., y Actis, W., (2003). 
Los seres humanos nos hemos enfrentado siempre al reto, teórico y práctico a la vez de aumentar nuestros conocimientos y de transformar la realidad circundante y así hemos ido acumulando saberes sobre el entorno en que vivimos. Este conjunto de conocimientos que las personas tenemos sobre el mundo, así como la actividad humana destinada a conseguirlo es lo que denominamos ciencia (derivada del latín "scire" que significa saber, conocer; su equivalente en griego es "sophia" que significa arte del saber). No obstante el título de ciencia no se puede aplicar a cualquier conocimiento sino únicamente a los saberes que han sido obtenidos mediante una metodología, y que cumplen con determinadas condiciones. Marques, (2003).

Zapata, (2014), la formación académica deberá ser integral, superando todo el lastre tradicional docentista, bajo una visión aglutinadora de las actividades académicas elaboradas a partir de la comprensión de que la esencia de la educación superior es la formación integral de las personas que acuden a ella. Si las instituciones de educación superior no forman de manera integral a sus alumnos, no cumplen su función ni su razón de ser.

La comprensión integral e integradora de la formación académica posibilitará la construcción de un proyecto educativo que sea capaz de formar un sujeto crítico, con capacidad de globalización y acción en circunstancias cambiantes y críticas. De lo contrario, la educación superior continuará ofreciendo egresados parcialmente formados, inseguros y sin un horizonte claro; profesionales que creen que el trabajo en la sociedad consiste solo en la aplicación de unas cuantas técnicas y recetas memorizadas, en un mal entendido proceso de formación. Zapata, (2014).

La formación para la investigación puede denominarse como un proceso que implica prácticas y actores diversos, en el que la intervención de los formadores como mediadores humanos se concreta en un quehacer académico consistente en promover y facilitar, preferentemente de

\footnotetext{
371 Vol. 4, núm. 3, julio 2018, pp. 360-391 Verónica R. Indacochea-González; Carmen A. Álvarez-Vásquez; José M. Piguave-Reyes; Marlon A. Cañarte-Chele; Virginia E. Pincay-Pin; Rosa del Rocío Pinargote-Chancay
} 
manera sistematizada (no necesariamente escolarizada), el acceso a los conocimientos y el desarrollo de habilidades, hábitos y actitudes, e internalización de valores, que demanda la realización de la práctica denominada investigación. Moreno, (2002)

La investigación educativa se ha constituido en una categoría conceptual amplia en el estudio y análisis de la educacion. Trata las cuestiones y problemas relativos a la naturaleza, epistemología, metodología y objetivos en el marco de la búsqueda progresiva del conocimiento en el ámbito educativo. (Latorre et al., 1996), citado por Carrasco y Calderero, (2000).

La investigación como base de la enseñanza y de formación permite al docente, desde la reflexión crítica, la construcción de saber dejando de lado el rol de mediador pasivo entre teoría y práctica. Salcedo, (2003).

El investigador social del siglo XXI, debe tratar a la comunidad como un equipo de trabajo, en el que no se prohíba establecer lazos afectivos, siempre y cuando se procure la objetividad de su labor. Por consiguiente, esta estrategia se recomienda en proyectos de carácter social y el trabajo en conjunto con las comunidades, pues son necesarias tanto para el empoderamiento de éstas hacia su patrimonio común, como para el cambio cognoscitivo y estructural de los estudiantes que salen del salón de clase. Así, la experiencia de vinculación se convierte en un medio de enlace entre la academia y la cultura popular, puesto que articula, enfrenta y pone a dialogar, diversas realidades: comunidad/ámbito institucional, cultura popular/cultura académica, conocimiento tradicional/conocimiento científico, los cuales no pueden articularse naturalmente. Maass y Sabulsky, (2015)

\footnotetext{
372 Vol. 4, núm. 3, julio 2018, pp. 360-391 Verónica R. Indacochea-González; Carmen A. Álvarez-Vásquez; José M. Piguave-Reyes; Marlon A. Cañarte-Chele; Virginia E. Pincay-Pin; Rosa del Rocío Pinargote-Chancay
} 
La investigación debe asumirse como una actividad cotidiana de los seres humanos y asunto medular es la motivación interna o externa para realizarla ya que su propósito fundamental es generar conocimientos. Flores y Pacheco, (1991).

La investigación como un proceso cuya finalidad consiste en propiciar y fomentar el interés y el espíritu creativo en un proceso de aprendizaje. En este sentido la investigación no se propone como intención primaria producir conocimientos nuevos, es más bien un procedimiento pedagógico. Morán, (1993).

En este contexto el compromiso de la universidad exige de su habilidad y efectividad para responder a las necesidades de transformación de la sociedad donde está inmersa, mediante el ejercicio de sus funciones básicas: docencia, investigación y extensión/proyección social. Estas funciones deben estar respaldadas por la búsqueda de la promoción de la justicia, la solidaridad y la equidad social, mediante la construcción de respuestas exitosas para atender los retos que implica promover el desarrollo humano integral Guillén, (2012).

Según, Ibarrola (1989), sería importante apoyar la conformación de equipos de investigación cuyos integrantes puedan todos experimentar el proceso completo de la investigación. Se insiste en procesos completos de investigación, esto es, de reflexión teórica unida a trabajo empírico, con lo que ello implica de respeto a los tiempos que dichas tareas requieren. Apoyo también para la presentación de resultados y la confrontación de los mismos, con fines formativos. Se insiste en el trabajo de equipo por oposición al de las organizaciones piramidales, que propician una aguda división del trabajo intelectual. El anterior no es un planteamiento de democracia populista, sino de mayor eficiencia en la formación de más y mejores investigadores.

\footnotetext{
373 Vol. 4, núm. 3, julio 2018, pp. 360-391 Verónica R. Indacochea-González; Carmen A. Álvarez-Vásquez; José M. Piguave-Reyes; Marlon A. Cañarte-Chele; Virginia E. Pincay-Pin; Rosa del Rocío Pinargote-Chancay
} 
En nuestros días se presenta una preocupación más o menos extendida de que las instituciones educativas, en lo general, no están cumpliendo a cabalidad con su responsabilidad de formar a las nuevas generaciones con las capacidades requeridas en una sociedad que se transforma profunda y aceleradamente (Chiappe \& Guido, 2009; Didriksson, s/f; Marcelo, 2001; Mella, 2003; Tedesco, 1996), citados por Pérez, (2012).

Cohen, (2007), la Universidad redefine su responsabilidad para establecer un equilibrio entre las necesidades reales y el lograr incidir en la transformación de la comunidad, acabando con la organización separada del saber en especialidades y, al contrario, instituir el pensamiento holístico, complejo, transdisciplinario y práctico para formar una comunidad de aprendizaje asociativo con calidad y pertinencia.

Bueno y Casini, (2007), expresan que, el futuro de la «tercera misión» dependerá del grado de armonía e integración de objetivos y comportamientos del marco político-gubernamental, del marco social y empresarial y del marco académico-investigador de la universidad y de los Organismos Públicos de Investigación. Sincronía o diacronía que puede ser la clave para el éxito o el fracaso de la propuesta de la «tercera misión», como nueva perspectiva de la función de la Universidad en la actual sociedad del conocimiento.

Esta integración y cooperación responde a la metáfora, antes citada, del «modelo de triple hélice» o mejor, en lenguaje técnico-aeronáutico, de una hélice de «triple pala», en la que el giro coordinado de cada una de las palas que representan los agentes que protagonizan los actuales Sistemas de Ciencia, Tecnología y sociedad, es decir, universidades, gobiernos y empresas, podrá generar la energía necesaria para que el sistema de innovación despegue y se eleve lo más alto posible. Dadas las inercias, la complejidad y los recursos y capacidades que se ven involucrados en

\footnotetext{
374 Vol. 4, núm. 3, julio 2018, pp. 360-391
Verónica R. Indacochea-González; Verónica R. Indacochea-González; Carmen A. Álvarez-Vásquez; José M. Piguave-Reyes; Marlon A. Cañarte-Chele; Virginia E. Pincay-Pin; Rosa del Rocío Pinargote-Chancay
} 
la definición y puesta en práctica con cierto éxito de la «tercera misión» de la Universidad para los próximos años. Bueno y Casini, (2007).

Los retos que estas manifestaciones plantean a la educación terciaria, están en marcha, en casi todas las regiones del mundo, procesos de transformación universitaria cada vez más profundos, que persiguen que la institución que llamamos "la Universidad" supere los nuevos retos y sobreviva, manteniendo incólume lo que ha sido hasta ahora su propia esencia. Así lo vislumbró la "Declaración Mundial sobre la Educación Superior para el Siglo XXI", aprobada en París en 1998, cuando señaló que para responder a tales desafíos, las universidades debían emprender "la reforma más radical que jamás antes hayan enfrentado". Tünnermann, (2011).

Vargas, R., (2015), expresa que, superando el sentido de capacitación en Investigación Educativa (IE) y transformando el proceso en un dispositivo de formación y autoformación, tanto para las y los asesorados de Educación Básica y Superior como para las y los responsables de los Programas de Superación Profesional del Departamento de Posgrado e Investigación (DPI). Para alcanzar los propósitos del Acompañamiento Formativo (AF), se implementa el procedimiento metodológico siguiente: promover un espacio grupal de interacción dialógica, a partir sobre todo de reflexiones heurísticas, así como el desarrollo de habilidades investigativas, entre ellas, el saber escuchar, afinar la mirada, la habilidad de escribir, la lectura exegética.

Que se practican al elaborar cada participante su Trayecto Formativo, así como, un Diario de Campo por sesión. Búsqueda y registro de información de manera sistemática y sobre todo sustentación teórica de cada uno de los pasos del proceso investigativo, todo ello es posible al desarrollarse las sesiones de un Seminario Taller de Formación en Investigación Educativa (STFIE),

\footnotetext{
375 Vol. 4, núm. 3, julio 2018, pp. 360-391 
involucrando la perspectiva democrática e inclusiva de la educación en la escuela pública, como también la investigación en los procesos de formación docente. Vargas, R., (2015).

La vinculación con la comunidad como estrategia de desarrollo

La vinculación como estrategia de desarrollo en las Universidades públicas. California: Instituto de Investigación y Desarrollo Educativo de la Universidad de Baja California. Se ha convertido en un aspecto muy importante de las políticas de desarrollo a nivel nacional y durante los últimos años han impulsado diversas acciones tendientes a reforzar estas actividades, Maldonado y Gould (2012).

Es imperante el diálogo de saberes académico y popular; para contribuir al "fin de la pobreza", ODS 2016-2030. El enfoque asumido se basa en la ética, el humanismo y las dinámicas participativas para propiciar cambios en las comunidades, para superar asistencialismo, dependencia y mentalidad de invalidez, por una dinámica de estudio, análisis y diálogo de los procesos históricos y actuales que posibiliten transformación y aprendizaje significativo, para erradicar la pobreza efectiva y consistentemente. Se propende porque el ethos de la universidad se base en su competencia comunicativa y de servicio para que la comunidad participe plenamente en su gestación y desarrollo. Hoyos, G. (2003). Citado por Parra de Marroquín, (2018).

Tünnermann, (2011), indica que los retos provenientes del conocimiento contemporáneo y de la sociedad global, es urgente estructurar las respuestas de las instituciones de educación superior. En primer lugar, y ante un mundo en constante proceso de cambio, la educación permanente aparece como la respuesta pedagógica estratégica que hace de la educación asunto de toda la vida y dota a los educandos de las herramientas intelectuales que les permitan "aprender a aprender" y

\footnotetext{
376 Vol. 4, núm. 3, julio 2018, pp. 360-391 
adaptarse a los nuevos requerimientos del mundo social y laboral, y a la expansión y obsolescencia del conocimiento.

Las universidades, están preparando a los estudiantes para enfrentarse a la sociedad. Los mismos manifiestan que "La universidad se enfrenta entonces al problema de cómo llevar a la práctica lo que se predica y cómo evaluar el efecto de las prácticas tradicionales en la transformación social y el desarrollo de la región y el país que las acoge”. Hernández y Saldarriaga, (2009).

López, (2018), indica que las actividades de vinculación y sus resultados pueden promover el desarrollo de nuevas líneas de investigación y la adecuación de la oferta académica a las demandas sociales. Pueden, así mismo, fortalecer el papel de la universidad en el debate social y en la construcción de una agenda pública en relación con las distintas problemáticas que afectan al país. De hecho, recuperar la actoría social de la universidad pública es una tarea pendiente. Para recuperar el liderazgo en el debate público se requiere fortalecer la imagen institucional de la universidad. Algo que no podrá lograrse si antes no se confrontan las expectativas de cambio con la realidad e identidad histórica de la universidad ecuatoriana.

Martínez, (2007), Expresa, que la promoción y divulgación de lo que hace la extensión es vital, hacer alianzas estratégicas con las empresas con otros organismos gubernamentales y no gubernamentales. Decirle a la sociedad, a la colectividad, esto es lo que hace la Universidad, esa es la contribución para el desarrollo regional y nacional, eso es extensión, buscar, conectarse con el entorno. 
La formación de recursos humanos de excelencia académica y profunda sensibilidad social constituye la razón de ser de la educación superior, y el encargo social que debe asumir para desarrollar las cualidades intelectuales, humanas, culturales, artísticas, políticas y espirituales del estudiante, en conjunción con la naturaleza y la sociedad, articulando en forma orgánica y dinámica la docencia, la investigación, la vinculación con la sociedad, la difusión y la gestión académica, donde se entrelace razón y liberación. Zapata, W., (2014).

Valera y Sánchez (2018), Desde los lineamientos teóricos escogidos y analizados se ha intentado mostrar la vinculación estrecha que hay entre RST, Nuevo Humanismo, Prácticas Socioeducativas y Buenas Prácticas como partes indisolubles y encadenadas de un paradigma emergente, el de la sostenibilidad, compromiso, participación, inclusión y equidad. En el marco de la internacionalización de la educación superior y desde el compromiso social territorial ineludible de las academias, iniciado hace un siglo en Córdoba de la mano de los estudiantes, visualizamos que se abre camino a una responsabilidad social internacional, como accionar entrelazado, socialmente responsable y comprometido con el ecosistema social, donde los intelectuales tenemos una misión indelegable.

la Universidad contribuye a reconocer dentro de estas necesidades lo que son las características, las culturas, las potencialidades necesarias y demandas que el medio esta cada día planteándole dispuesta a abrir múltiples y flexibles formas de interacción con esos sectores para darles también respuestas oportunas, deberá estar abierta y promoverá espacios de cooperación con otros centros, con otras universidades, con otros espacios, para que estas respuestas puedan ser un poco más congruentes y mucho más fortalecidas a través de las acciones que se ejercen. Martínez, (2007)

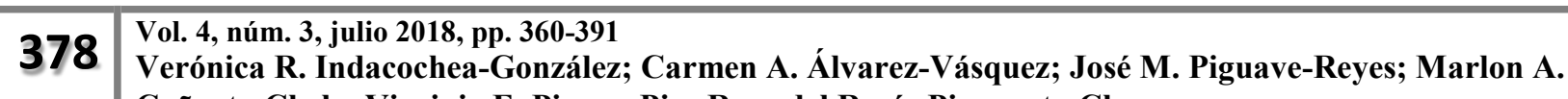
Cañarte-Chele; Virginia E. Pincay-Pin; Rosa del Rocío Pinargote-Chancay
} 
Blanco y Nobrega (2016), afirman que, el docente comunitario viene a ser una especie de coordinador o maestro de la orquesta, en cual hay personas con distintos instrumentos y su trabajo constituye la armonización, la canalización y la organización para la ejecución de una melodía. Así es la función del docente universitario en el trabajo comunitario.

\section{La vinculación con la sociedad y los sectores productivos}

En relación con la IES, cuyo caso nos interesa presentar, los profesores e investigadores han elaborado estrategias y llevado a cabo acciones para establecer relaciones de vinculación con el sector productivo acordes con su misión, como universidad pública y sin menoscabo de su identidad. Ello ha sido posible no sólo a través del conocimiento y capacidades de la comunidad de esta institución educativa y de la formación de redes internas de colaboración, sino también en acuerdo con su modelo educativo y con los propósitos de formar a sus alumnos como profesionales conocedores y solidarios con los problemas de la realidad económica y social. Oropeza et al., (2014).

Las universidades deben abrir sus puertas a todas las personas hábiles interesadas en la adquisición de conocimiento y ofrecer respuestas oportunas y de calidad a los intereses y necesidades de los alumnos. Al configurar su oferta de servicios y los programas institucionales de vinculación, las IES deben reconocer las necesidades de los sectores sociales y productivos. Además, se deberá superar la concepción de la vinculación como un camino de una sola vía (oferta de servicios) y buscar estrategias que conduzcan al establecimiento de programas en que ambas partes obtengan beneficios valiosos, diferentes y complementarios. Botello de León, M., (2012).

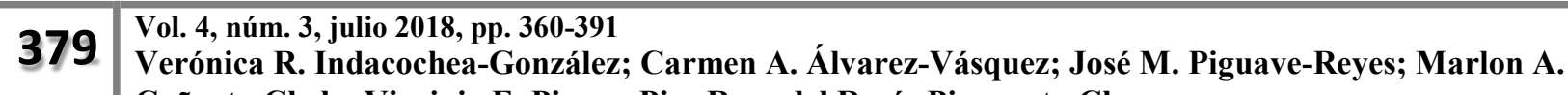
Cañarte-Chele; Virginia E. Pincay-Pin; Rosa del Rocío Pinargote-Chancay
} 
La vinculación con los diversos sectores productivos puede ser una actividad muy importante que redimensione las funciones sustantivas de la universidad, docencia, investigación y extensión. Sin embargo, estas acciones deberán evaluarse a fin de que permitan retroalimentar estas actividades y mejorarlas. Maldonado y Gould (2012).

Se busca compartir buenas prácticas universitarias posibles de ser replicadas por otras universidades dentro de Latinoamérica y el Caribe, desde lo que entendemos por RST. Por otra parte, a partir del trabajo colaborativo entre academias de distintos países, de su intercambio y enriquecimiento mutuo, hemos considerado la posibilidad de realizar aportes al concepto de RST en su dimensión internacional, teniendo en cuenta sus vínculos, las redes en las que se participa y la necesaria y urgente responsabilidad social que se requiere frente los problemas de índole mundial como el calentamiento global, la contaminación ambiental y la concentración económica lacerante, con el consecuente crecimiento exponencial de la pobreza, producto de un orden social injusto que requiere de un accionar transnacional, comprometido y responsable. Valera y Sánchez (2018).

La Vinculación con la Sociedad orienta los procesos de Prácticas pre-profesional y proyectos comunitarios, considera a la vinculación como el vínculo permanente que establece esta unidad académica con los sectores comunitarios, productivos, culturales e instituciones de servicios, en función de generar el conocimiento mediante una acción sistemática de aprender y enseñar, que, hacia adentro, fortalece el perfil profesional de los estudiantes, enriquece el acervo del docente y hacia fuera contribuye a la solución de problemas educativos y sociales. Simbaña, (2017).

Botello de León, M., (2012), las evidencias reales señalan que, aún en los casos de las universidades más desarrolladas de nuestro país como la Universidad Nacional Autónoma de México o la Universidad Autónoma Metropolitana-Iztapalapa, con una base de investigación

\footnotetext{
Vol. 4, núm. 3, julio 2018, pp. 360-391
Verónica R. Indacochea-González Verónica R. Indacochea-González; Carmen A. Álvarez-Vásquez; José M. Piguave-Reyes; Marlon A. Cañarte-Chele; Virginia E. Pincay-Pin; Rosa del Rocío Pinargote-Chancay
} 
altamente consolidada, en las cuales la vinculación con el sector industrial y público han cristalizado importantes convenios y contratos de asesoría, aun así en esos centros de estudios la vinculación no ha alcanzado la madurez y el grado de desarrollo que se refleje en todo el funcionamiento general de las instituciones.

La perspectiva productivista, entiende a la vinculación como válida, sólo si la realiza el sector productivo de la economía y más específicamente la estructura industrial, atendiendo problemas tecnológicos del sector productivo. En este sentido, la vinculación señala un proceso de transferencia de tecnologías que puede implicar el establecimiento de puentes entre la investigación científica y el desarrollo tecnológico, para atender problemas del entorno; creando pequeñas unidades de transferencia de tecnología al interior de cada dependencia. Botello de León, M., (2012).

Brünner, (2002) y Tedesco, (2000), coinciden en señalar que, a la par del veloz desarrollo de las tecnologías va tomando fuerza la idea de educación permanente y capacidad adaptativa al trabajo, lo que implica que será necesario educarse a lo largo de toda la vida para poder adaptarse a los requerimientos cambiantes del desempeño social y productivo.

Tedesco, J., (2000), "Educar en la sociedad del conocimiento”, Fondo de Cultura Económica, Buenos Aires.

El proceso de transferencia de conocimiento tecnológico generado en el ámbito universitario hacia las empresas públicas y privadas ha sido objeto de atención de muchos investigadores en los últimos años, para ello han tenido en cuenta variables endógenas y exógenas de amplio alcance tanto

\footnotetext{
381 Vol. 4, núm. 3, julio 2018, pp. 360-391 Verónica R. Indacochea-González; Carmen A. Álvarez-Vásquez; José M. Piguave-Reyes; Marlon A. Cañarte-Chele; Virginia E. Pincay-Pin; Rosa del Rocío Pinargote-Chancay
} 
como los mecanismos particulares que los agentes implementan y el producto mismo objeto de la transferencia. OCDE, (1994).

OCDE (1994). Main Definitions and conventions for the measurement of research and experimental development (RyD). A sumary of the Frascati Manual. Organisation for economic cooperation and development. Paris.

La educación superior debe orientar la docencia a la formación de profesionales emprendedores que respondan proactivamente a las necesidades de los sectores productivos; que promocionen la investigación técnica y aplicada, y promuevan las vinculaciones con la comunidad, en un contexto guiado por valores éticos, la orientación a la calidad académica y al servicio comunitario. Zapata, W., (2014).

La responsabilidad social universitaria es saber administrar los impactos que la universidad tiene en sus cuatro áreas de funcionamiento (administración, formación, investigación y participación social). Así es necesario que sea responsable no solo de cara hacia su entorno a través de proyectos sociales que incluyen a estudiantes de varias especialidades, sino también en la manera en la que, por ejemplo, recicla sus desechos, rinde cuentas transparentes o cuida del personal administrativo y académico. No es sólo asegurar que sus estudiantes sean el día de mañana responsables, es también asegurarse de que ella misma lo es. Ayala, M., (2011).

Ayala G., Mauricio O., (2011), Responsabilidad social universitaria, $<$ http://www.redicces.org.sv/jspui/bicscream/10972/91/1/Responsabilidad\%20social\%20 universitaria.pdf $>$

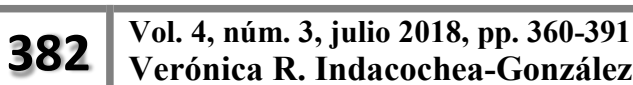
Verónica R. Indacochea-González; Carmen A. Álvarez-Vásquez; José M. Piguave-Reyes; Marlon A. Cañarte-Chele; Virginia E. Pincay-Pin; Rosa del Rocío Pinargote-Chancay
} 
Silva, (2006), la nueva formación profesional que esta modalidad educativa recoge es la vinculación escuela-empresa, con la finalidad de combinar los conocimientos adquiridos en el salón de clases con la práctica o el "aprender a hacer" en un centro de trabajo. La alternancia entre la escuela y el sector productivo permite a los estudiantes conocer el contexto real del trabajo e integrar conocimientos teóricos, tecnológicos y organizativos, lo que facilitará su posterior ingreso al mundo del trabajo.

Silva, M., (2006), "La calidad educativa de las universidades tecnológicas: Su relevancia, su proceso de formación y sus resultados”, ANUIES, México.

Fabara G., E., (2016), uno de los aspectos que debería tomarse en cuenta en una posible normativa para el futuro es la necesidad de incorporar más obligaciones y responsabilidades en el campo de la vinculación con la colectividad, puesto que los docentes universitarios del Ecuador deben tener una amplia conciencia social que permita a la institución educativa contribuir al mejoramiento de las condiciones de vida de los ciudadanos. A pesar de los esfuerzos realizados, en el país aún hace falta fortalecer una cultura nacional a favor de la educación superior e instaurar una profesión académica para el ejercicio de la docencia universitaria, con todas las implicaciones que tiene el desarrollo de una disciplina necesaria para la construcción del nuevo país.

Fabara G., Eduardo, (2016). La formación y el ejercicio de la docencia universitaria en Ecuador. Desafíos. Alteridad, 11(2), pp. 171-181.

El Artículo Noveno del Reglamento de Carrera y Escalafón del Profesor e Investigador del Sistema de Educación Superior establece las principales responsabilidades y funciones docentes, las cuales se concentran en los siguientes aspectos, CES, (2012).

\footnotetext{
383 Vol. 4, núm. 3, julio 2018, pp. 360-391 
10. "Diseño e impartición de cursos de educación continua o de capacitación y actualización;" Las instituciones de educación superior deben ser consideradas siempre como “organizaciones que aprenden”, en las que todos sus miembros deben fortalecer permanentemente su talento humano. Más aún se deben promover oportunidades de crecimiento y desarrollo para todos quienes forman las entidades educativas. La organización de eventos para la comunidad universitaria y para la colectividad cercana o afín a los propósitos de la institución es un trabajo permanente, que no debe dejar de realizarse siempre.

11. "Participación en actividades de proyectos sociales, artísticos, productivos y empresariales de vinculación con la sociedad articulados a la docencia e innovación educativa;" La universidad del siglo XXI no debe estar enclaustrada en sus cuatro paredes, debe hacer parte de los proyectos que se gestan al interior de la comunidad, debe promover la realización de proyectos de mejoramiento de las condiciones de vida, debe apoyar las iniciativas para fortalecer las organizaciones sociales y debe contribuir con sus conocimientos y experticia en la solución de los problemas ambientales, económicos, políticos y sociales.

CES, (2012), "Reglamento de Carrera y Escalafón del Profesor e Investigador del Sistema de Educación Superior". Quito.

López M., F., (2018), La vinculación obligatoria de la investigación al cambio de matriz productiva y a los planes de desarrollo se refuerza con el peso que, en el modelo de universidad que guía la reforma, se le asigna a la función de vinculación con la sociedad. Inspirada en la reforma de Córdova de 1918, que sustentó la creación de las denominadas extensiones de universitarias, la vinculación con la sociedad se ha convertido en una función de la universidad ecuatoriana tan importante como las funciones de docencia e investigación social. 
Se hace indispensable, de otro lado, reconociendo que las funciones esenciales de la universidad son la docencia y la investigación, redimensionar y reformular el alcance de vinculación con la sociedad, y situarla en el sitio que le corresponde. Es decir, no como una función autónoma y de igual importancia que la docencia y la investigación, sino como un mecanismo para reforzar tanto la una como la otra. López M., F., (2018).

Brunner, (2003), manifiesta que, la educación ha estado siempre estrechamente imbricada con la tecnología. Luego, así como se habla de las bases tecnológicas de un modo de producción por ejemplo del modo de producción industrial- es posible, asimismo, hablar de las bases tecnológicas de la producción educacional. A fin de cuentas, la educación -como empresa social- es también una producción: la producción de un tipo humano determinado culturalmente o, si se quiere, la producción de unas competencias, unas disciplinas y unos conocimientos que necesitan ser comunicados e inculcados. Desde el punto de vista de la sociedad, se trata de la producción y reproducción del capital cultural distribuido entre individuos, familias, grupos y clases.

Brunner, J. J., (2003), Las nuevas tecnologías y el futuro de la educación, “La educación al encuentro de las nuevas tecnologías”, UPE UNESCO / Septiembre Grupo Editor, Argentina.

Se trabaja en la construcción de estrategias para el mejor cumplimiento de sus objetivos en la investigación, la docencia y el servicio a la sociedad en su vinculación con empresas; estrategias que permitan ampliar el impacto de los trabajos de investigación, de los propios investigadores y de los alumnos guiados por éstos, en la atención y solución de problemas empresariales. Oropeza et al., (2014). 
El rol de las universidades en la sociedad de la información adquiere una importancia destacada en la transferencia de los avances científicos y tecnológicos al medio socio-productivo en que se asientan, a través de las diversas actividades de vinculación con que interactúan las organizaciones educativas y las del medio socio-productivo. En su relación con el medio socioproductivo, las instituciones de educación superior han empleado diferentes estrategias y modalidades a efectos de transferir y promocionar los avances científicos y tecnológicos en las actividades socio-productivas y promover el interés y la participación del personal académico en dichas actividades. Allende, Gonzalez, y Zanfrillo, (2010).

Allende, Olivia, Gonzalez, María y Zanfrillo, Alicia, (2010), Un estudio de estrategias de vinculación universidad y entorno socio-productivo, Temas de Ciencia y Tecnología vol. 14 número 42, pp $41-52$

\section{Conclusiones.}

En la actualidad la Educación superior frente a, le ha provocado un continuo y acelerado cambio, esto implica un gran desafío para la universidad, ya que con los nuevos escenarios y demandas sociales debe responder ante la comunidad interviniendo y transformando su entorno marcando su desarrollo. Los procesos de globalización han generado cambios en la Institución de Educación Superior (IES), la vinculación como estrategia frente la formación de profesionales por competencias.

Las universidades tienen un reto importante puesto que son ellas las que forman a los científicos, profesionales y líderes que decidirán de las políticas económicas y públicas nacionales e internacionales. La estructura académica debe generar una mejor calidad educativa orientada a

\footnotetext{
386 Vol. 4, núm. 3, julio 2018, pp. 360-391 
competir. La vinculación con la comunidad como estrategia de desarrollo, es la condición más importante como políticas de desarrollo a nivel nacional.

Durante los últimos años se han impulsado diversas acciones tendientes a reforzar estas actividades se aconseja como estrategia elaborar proyectos de carácter social y el trabajo en conjunto con las comunidades. La universidad debe responde al "replanteamiento" de la política social incorporando en sus programas de estudios la Investigación Acción Participativa (IAP), destinada al trabajo social y al desarrollo comunitario.

La vinculación con la sociedad y los sectores productivos apoyado por los sectores estatales considerando la importancia de la ciencia, la tecnología y la innovación, los tutores e investigadores han de elaborar estrategias y propender tareas para establecer relaciones de vinculación con el sector productivo; se vuelve importante incorporar programas, con una estrategia general de intervención que se permita plasmar en muchas prácticas educativas hacia las necesidades de la realidad social y productiva, todo esto en el ámbito en que se desenvuelve la universidad con los sectores sociales, productores y estudiantes en formación profesional, que la universidad investigue la necesidad de vinculación que tiene con el entorno globalizado.

El futuro de la universidad se fundamenta en la mejora continua, en la calidad de sus procesos, en la capacidad que tenga para repensar e innovar su aporte ante una sociedad cambiante, la IES debe dar respuestas estratégicas a través de la vinculación para el desarrollo lo que permite generar actividades en la que los estudiante puedan aprender juntos, es decir los participantes sean estos los educandos como los beneficiarios de determinado proyecto.

\footnotetext{
387 Vol. 4, núm. 3, julio 2018, pp. 360-391 Verónica R. Indacochea-González; Carmen A. Álvarez-Vásquez; José M. Piguave-Reyes; Marlon A. Cañarte-Chele; Virginia E. Pincay-Pin; Rosa del Rocío Pinargote-Chancay
} 


\section{Referencias Bibliográficas}

Alcántar, V. y Arcos, J., (2004), La vinculación como instrumento de imagen y posicionamiento de las instituciones de educación superior. Revista Electrónica de Investigación Educativa, 6(1). http://redie.uabc.mx/vol6no1/contenido-enriquez.html

Álvarez G., J., Romero F., A., Murillo P., D., (2014), Estrategia para la formación de competencias profesionales en la carrera de Administración de Empresas de la UNIANDES. Revista Avanzada Científica ISSN 1029-3450, Vol. 17 No. 3.

Barabtarlo y Z., (2002), Investigación cualitativa y Formación de Profesores: Diario de Campo e Historia de vida, En Revista: El Despertar Académico. Universidad del Valle de México. Año 4, No. 17.

Bellettini, O., (2012), Los centros de políticas públicas y la sociedad del conocimiento en América Latina, Grupo Faro

Blanco y Nobrega (2016), Blanco, C., \& Nobrega, E. (2016). Rol estratégico de los docentes universitarios en el servicio comunitario. Revista Ciencias de la Educación.

Botello de León, Minerva (2012). La vinculación como estrategia para el desarrollo académico del Diseño Industrial, Tesis de Maestría en la FARQ UANL.

Bueno, E. y Casani F., (2007), "La tercera misión de la universidad. Enfoques e indicadores básicos para su evaluación", en Economía Industrial, núm. 366, pp. 43-59.

Briones, G., (1999), La investigación social y educativa, Tercer Mundo, Colombia.

Brünner, José Joaquín, (2002), “Educación e Internet ¿La próxima revolución?”, Fondo de Cultura Económica, Chile.

Carrasco, J., y Calderero, J., (2000), Aprendo a investigar en educacion, Ediciones RIALP S.A., España.

Cohen, Daniel, (2007), Desafíos de la Responsabilidad Social Universitaria. Razón y Palabra [en linea]: consulta: 18 de julio de 2018] Disponible en: $\leq$ http://www.redalvc.org/articulo.oa?id $=\mathbf{1 9 9 5 2 0 7 3 5 0 2 3}>$ ISSN 1605-4806

Costa, V., (2011), Formação de professores e educação inclusiva: experiências na escola pública, En: Costa, V., Carvalho, M., Miranda, T., Damasceno, A., (Org.), Políticas públicas e produção do conhecimento em educação inclusiva, INTERTEXTO-CAPES, p. 31-52, Niterói, Rio de Janeiro.

Costa, V., (2012), Políticas públicas de educação: demandas e desafíos à inclusão na escola pública, En: Mendes, E., 7 Almeida, M., (Org.), a pesquisa sobre inclusão escolar em suas múltiplas dimensões: teoría, política e formação, Coleção Inclusão Escolar, vol.1, ABPEE, p. 107-125, Marília, São Paulo.

Ferry, G., (1997), Pedagogía de la formación, Editorial Novedades Educativas, Argentina. p. 56

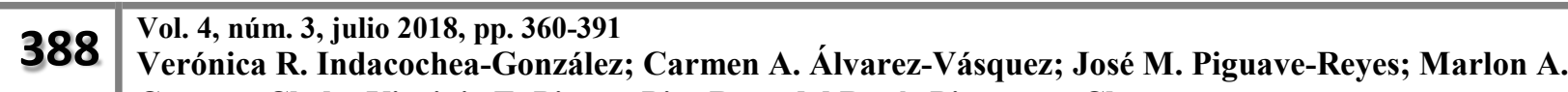
Cañarte-Chele; Virginia E. Pincay-Pin; Rosa del Rocío Pinargote-Chancay
} 
Flores, I., y Pacheco, A., (1991), La investigación una forma de aprender, México.

GRIMALDO, Humberto, (2018), La Responsabilidad Social Territorial: aprendizaje, armonización y transformación. Revista Educación Superior y Sociedad (ESS) ISSN: 0798-1228 / ISSN: 2610-7759 (En línea), [S.1.], v. 26, n. 26, p. 175-199, Disponible en: $<$ http://ess.iesalc.unesco.org.ve/ess3/index.php/ess/article/view/583>.

Guillén, L., (2012), "La responsabilidad social universitaria: un estudio de caso aplicado a la Universidad Autónoma del Caribe en Barranquilla, Colombia", en Memorias VIII Congreso Internacional de Educación Superior Universidad 2012, La Habana, Cuba.

Hernández, R., y Saldarriaga, A., (2009), Gestión de la responsabilidad social universitaria, Caso: Escuela de Ingeniería de Antioquia-EIA, DYNA, Medellín.

Ibarrola, M., (1989), La formación de investigadores educativos en México, en Universidad Futura, UAM - Azcapotzalco, Vol. 1, № 3, p.12-26, México.

López, F., (2018), Los retos de la educación universitaria en el Ecuador, www.planv.com.ec /historias/sociedad/retos-la-educacion-universitaria-el-ecuador

Maldonado, L y Gould, G. (2012). La vinculación como desarrollo en las universidades públicas. 11.

Maass, M. y Sabulsky, G. (2015), La vinculación como estrategia de formación en la educación superior, RED INNOVA CESAL. http://www.innovacesal.org/micrositio_redic_2015/05_redIC_HCS_ADA_guia_ed.pdf

Martínez de Carrasquero, C., (2007), "La responsabilidad social universitaria como estrategia para la vinculación con su entorno social", Brasil, Virtual Educa.

Marques, P., (2003). Reflexiones sobre la ciencia, el conocimiento y el método científico. http://peremarques.pangea.org/uabcienc.htm

Morán, O. P., (1993), La vinculación docencia: investigación como estrategia pedagógica. En: Revista Perfiles Educativos, Nº 61, México.

Moreno, B., (2002), Formación para la investigación centrada en el desarrollo de habilidades, Ediciones Universidad de Guadalajara, México.

Navia, A., (2006), Autoformación de maestros en los márgenes del sistema educativo. Cultura, experiencia e interacción formativa, Ediciones Pomares, México.

OEI, (2012), Ciencia, tecnología e innovación para el desarrollo y la cohesión social, OEI, Madrid, España.

Oseguera, M., (2005), Diagnóstico sobre políticas de investigación en el caso de Honduras, CSUCA/IESAL/UNESCO, Honduras. 
Oropeza Herrera, S., Peñalva Rosales, L. P., Pomar Fernández, S. \& Ruiz Lang, G. (2014). Estrategias de colaboración para fortalecer la vinculación de la universidad en apoyo a las Mipymes. Acta Universitaria, 24(NE-1), 37-47. doi: 10.15174/au.2014.707

Parra de Marroquín, O., (2018), Transformaciones sociales a partir del diálogo de saberes y el trabajo colaborativo entre las comunidades universitaria y local. Revista Educación Superior y Sociedad (ESS) ISSN: 0798-1228 / ISSN: 2610-7759 (En línea), [S.1.], v. 26, Nº. 26, p. 153-173, Disponible $<\underline{\text { http://ess.iesalc.unesco.org.ve/ess3/index.php/ess/article/view/582 > }}$. Fecha de acceso: 28 aug. 2018

Pereda, C., De Prada M. Á., y Actis, W., (2003), Investigación acción participativa: propuesta para un ejercicio activo de la ciudadanía, C/ Luna, $11-1^{\circ}$ dcha. 28004 Madrid.

http://www.psicosocial.net/grupo-accion-comunitaria/centro-de-documentacion-gac/trabajopsicosocial-y-comunitario/herramientas-investigacion-accion-participante/502-investigacionaccion-participativa-propuesta-para-un-ejercicio-activo-de-la-ciudadania/file

Pérez R., Abel, (2012), "Desigualdad, mercado laboral y educación superior en América Latina", Universidad Autónoma Metropolitana Unidad Azcapotzalco, El Cotidiano, núm. 176, México.

Salcedo, R., (2003). La investigación en el aula: y la innovación pedagógica, Biblioteca virtual Luis Ángel http://www.banrepcultural.org/blaavirtual/educacion/expodocen/expodocen8a.htm

Arango,

Sánchez, G., (1998), Fundamentos para la investigación educativa: presupuestos epistemológicos que orientan al investigador, Editorial Magisterio, Colombia.

Simbaña, H. (2017). Las implicaciones pedagógicas de la vinculación con la sociedad en la formación docente de los estudiantes de la Facultad de Filosofía, Letras y Ciencias de la Educación de la Universidad Central del Ecuador. Alicante.

Tünnermann Bernheim, Carlos, (2011), "La educación superior frente a los desafíos contemporáneos", Universidad Centroamericana, Nicaragua.

Valera, M., y Sánchez M., (2018), Buenas prácticas en universidades latinoamericanas y caribeñas Aportes al concepto de Responsabilidad Social Internacional Caso: UNCUYO (Argentina) y UNA (Costa Rica). Revista Educación Superior y Sociedad (ESS), ISSN: 0798-1228 / ISSN: 2610-7759 (En línea), [S.1.], v. 26, n. 26, p. 15-41, Disponible en: $<$ http://ess.iesalc.unesco.org.ve/ess3/index.php/ess/article/view/575>.

Vargas S., Raúl, (2015), Acompañamiento Formativo: Una Estrategia para la Formación en Investigación Educativa de Directivos, Docentes y Asesores de Educación Básica y Superior, revista entreideias, v. 4, n. 1, p. 35-49, Salvador.

Yurén, T., (2005), Ethos y autoformación del docente: análisis de dispositivos de formación de profesores, Editorial Pomares, México. 


Zapata, W., (2014), “Principales desafíos de la educación superior ecuatoriana”,
EcuadorUniversitario.Com

